\title{
Parents' Behaviors and Experiences Associated with Four Vaccination Behavior Groups for Childhood Vaccine Hesitancy
}

\author{
Morgan E. Ellithorpe ${ }^{1}$ [D $\cdot$ Robyn Adams ${ }^{2} \cdot$ Fashina Aladé $^{2}$
}

Accepted: 26 November 2021 / Published online: 7 January 2022

(c) The Author(s), under exclusive licence to Springer Science+Business Media, LLC, part of Springer Nature 2021

\begin{abstract}
Objectives Increasing vaccine hesitancy and decreasing acceptance of the Centers for Disease Control and Prevention's (CDC) recommended schedule for childhood vaccines represent a crucial public health issue. The present study directly compares vaccine acceptance behavior across four different groups: those who are fully accepting of the CDC-recommended schedule, those who are accepting but on a delayed schedule, those who only partially vaccinate, and those who do not vaccinate at all.

Methods A total of 779 adults residing in the United States with at least one child under the age of 18 years participated in an online survey.

Results Logistic and Ordinary Least Squares regression analyses revealed clear differences between the vaccination behavior groups on a variety of demographic, psychographic, and behavioral metrics. Results suggest financial and insurance-related barriers still hinder full vaccination, and there are differences by race, ethnicity, and educational attainment. Sources of information about vaccines also differed by vaccination behavior group, with those who never vaccinate more likely to rely on friends and family for information. Finally, those whose child experienced what the parent interpreted as an adverse reaction to a previous vaccine, even if that reaction was within the bounds of "normal", were more likely to report they delay or partially vaccinate.

Conclusions for practice These results have implications for public health policy and intervention campaigns, in particular that two-step flow campaigns and increased knowledge of normal vaccine side effects may ameliorate some vaccine hesitancy.
\end{abstract}

Keywords Vaccine hesitancy · Information sources · Childhood · Parents

\section{Significance}

What is already known on this subject Vaccine hesitancy is a challenge, and we replicated patterns of demographics and beliefs that are associated with vaccine acceptance.

Morgan E. Ellithorpe

mellitho@udel.edu

Robyn Adams

adamsr15@msu.edu

Fashina Aladé

aladefas@msu.edu

1 Department of Communication, University of Delaware, 125 Academy St., Newark, DE 19716, USA

2 Department of Advertising \& Public Relations, Michigan State University, 404 Wilson Rd., East Lansing, MI 48824, USA
What this study adds A comparison of predictors of vaccine acceptance across multiple distinct vaccination groups. The addition of sources of information and child prior behavior as less-studied variables.

\section{Introduction}

Low vaccination rates in the U.S. are associated with outbreaks of serious preventable diseases (Greenlee \& Newton, 2018; Phadke et al., 2016). In recent years, there has been a large increase in non-acceptance of the Centers for Disease Control and Prevention's (CDC) recommended childhood vaccination schedule (Dubé et al., 2013; Ventola, 2016). Only three-quarters of U.S. children receive all childhood vaccines at the recommended times that are considered ageappropriate (Kurosky et al., 2016). 
There has been a great deal of research attempting to elucidate the reasons for vaccine hesitancy. Structural barriers such as financial barriers and access to doctors and health centers reduce likelihood of following the complete childhood vaccination schedule (Davis et al., 2003; Hill et al., 2018). Individual-level barriers, such as one's belief system or religious views, tend to cut across socio-demographic groups (Dubé et al., 2013; Gust et al., 2008; Larson et al., 2014; McKee \& Bohannon, 2016; Sadaf et al., 2013; Smith et al., 2011; Yaqub et al., 2014). Beliefs about vaccines have been studied extensively in a variety of contexts, including the role of information and misinformation in vaccine acceptance behavior (Dubé et al., 2013; Gust et al., 2008; Larson et al., 2014; McKee \& Bohannon, 2016; Sadaf et al., 2013; Smith et al., 2011; Yaqub et al., 2014). One systematic review of the literature identified four major belief categories: religious reasons, personal beliefs, safety or health concerns, and desire for information from health care providers (McKee \& Bohannon, 2016).

Black and Hispanic children are less likely than non-Hispanic white children to have received all recommended vaccinations on time (Hill et al., 2020); however, Black Americans are less likely than non-Hispanic white Americans to choose an alternate schedule (de Cantuária Tauil et al., 2016; Dempsey et al., 2011). Additionally, lower income is associated with a lower likelihood of complete and on-time vaccination (Bryant et al., 2006; Temoka, 2013; Ventola, 2016). However, clusters based specifically on personal belief exemptions are increasing in predominantly non-Hispanic white and high-income schools (Carrel \& Bitterman, 2015; Morrison et al., 2020).

The pediatrician remains the most-sought source of information about vaccines, even among the vaccine-hesitant (Chung et al., 2017; Giambi et al., 2018). However, those who are vaccine-hesitant are likely to seek out other, informal sources of interpersonal information such as family and friends in their social networks (Chung et al., 2017; GesserEdelsburg et al., 2017). What is less clear is whether differences exist in reliance on informal interpersonal sources by type of vaccine hesitancy. One study found no difference between parents who refused all vaccines and parents that were vaccine hesitant in the likelihood of discussing vaccines with family (Gesser-Edelsburg et al., 2017). However, more research is needed to further understanding in this arena.

One of the ways that anti-vaccination campaigns and websites reach vaccine hesitant parents is through online narratives of extreme child vaccine reactions (Shelby \& Ernst, 2013). In addition, previous work has found that knowing a parent whose child had a severe reaction to a vaccine is associated with vaccine refusal and delayed vaccine schedules (Chung et al., 2017). However, previous experience with one's own child as a predictor of partial and delayed vaccination has not been examined. Side effects of vaccinations are common, though usually mild (Prevention, 2019). Parents who report their child experienced a negative reaction to a previous vaccination may be more likely to move to a partial or delayed vaccination schedule. Whether this reaction was actually in line with a common mild reaction or not is less material than the parents' interpretation of that response. The present study looks at parents' perceptions of previous child responses to vaccination as one potential reason for hesitancy in the future.

Most of this previous research either examines vaccine acceptance linearly (e.g., how many vaccines accepted), or focuses on one type of hesitancy compared to complete vaccination (e.g., delayed compared to regular schedules, those who specifically elect not to accept the varicella vaccine compared to those who accept the varicella vaccine, the anti-vaccination movement compared to those who fully vaccinate, etc.). The present study uses a national sample of parents in the United States to directly compare multiple classifications of parents based on their prior vaccination acceptance-never, partial, delayed, and complete vaccination.

\section{Method}

\section{Participants}

Details for sample demographics and central tendency information for all measures are presented in Table 1. Participants were 779 adults residing in the United States with at least one child under the age of 18 years. We oversampled participants who reported deviating from the CDC-recommended vaccination schedule. Participants with multiple children were instructed to report on the child with the most recent birthday, in order to randomly select from their children in terms of demographics. Data were collected from February 22, 2019 to May 24, 2019.

\section{Procedure}

The study procedure was conducted in accordance with the principles of the Declaration of Helsinki and was determined exempt by the Institutional Review Board at [University]. Participants were recruited for an online survey through survey panel company Dynata's opt-in national panel. After providing informed consent, respondents were asked demographic questions. They were then asked about their past vaccination decisions, their child's reactions to past vaccinations, and their discussions with sources of information about vaccines. They were also asked to describe their discussions with their child about vaccination (the results of this qualitative analysis are detailed in a separate article). 
Table 1 Central tendency information for all measures. Sample total $n=779$

\begin{tabular}{|c|c|c|}
\hline & $M$ or $n$ & $S D$ or $\%^{1}$ \\
\hline \multicolumn{3}{|l|}{ Demographics } \\
\hline \multicolumn{3}{|l|}{ Child sex } \\
\hline Female & 351 & 45.1 \\
\hline Male & 401 & 51.4 \\
\hline Other & 27 & 3.5 \\
\hline Child age & 9.1 & 5.4 \\
\hline \multicolumn{3}{|l|}{ Relationship to child } \\
\hline Parent & 715 & 91.8 \\
\hline Grandparent/other & 64 & 8.2 \\
\hline \multicolumn{3}{|l|}{ Participant sex } \\
\hline Female & 564 & 72.4 \\
\hline Male & 184 & 23.6 \\
\hline $\begin{array}{l}\text { Non-binary/Own terminology/Prefer not to } \\
\text { disclose }\end{array}$ & 31 & 4.0 \\
\hline Participant age & 38.02 & 11.5 \\
\hline \multicolumn{3}{|l|}{ Participant race/ethnicity } \\
\hline White & 511 & 65.6 \\
\hline Black & 82 & 10.5 \\
\hline Asian & 52 & 6.7 \\
\hline Hispanic & 43 & 5.5 \\
\hline Multiracial & 37 & 4.8 \\
\hline Other/Prefer not to disclose ${ }^{2}$ & 54 & 6.9 \\
\hline \multicolumn{3}{|l|}{ Education } \\
\hline High school or less & 162 & 20.8 \\
\hline Some college/trade/associate degree & 256 & 32.9 \\
\hline College degree & 188 & 24.1 \\
\hline Advanced degree & 131 & 16.8 \\
\hline \multicolumn{3}{|l|}{ Income } \\
\hline Less than $\$ 50,000 /$ year & 302 & 38.8 \\
\hline$\$ 50,000$ to $\$ 109,999 /$ year & 287 & 36.8 \\
\hline$\$ 110,000$ or more/year & 143 & 18.4 \\
\hline Health insurance (yes) & 704 & 90.4 \\
\hline \multicolumn{3}{|l|}{ Child vaccination measures } \\
\hline \multicolumn{3}{|l|}{ Vaccination status } \\
\hline Full vaccination & 420 & 53.9 \\
\hline Delayed vaccination & 134 & 17.2 \\
\hline Partial vaccination & 103 & 13.2 \\
\hline No vaccination & 122 & 15.3 \\
\hline Consult Pediatrician (yes) & 577 & 74.1 \\
\hline Follow Ped. Advice (yes) & 485 & 84.6 \\
\hline \multicolumn{3}{|l|}{ Sources of information (yes) } \\
\hline Spouse/partner & 526 & 67.5 \\
\hline Parents & 157 & 20.2 \\
\hline Other family & 64 & 8.2 \\
\hline Friends & 76 & 9.8 \\
\hline \multicolumn{3}{|l|}{ Child response to vaccines } \\
\hline$(1-5$ scale $; n=657)$ & 2.37 & 1.07 \\
\hline Change in intention ( $1-5$ scale $)$ & 2.82 & 0.76 \\
\hline
\end{tabular}

${ }^{1}$ Total percent may not sum to $100 \%$ in each category due to missing values and rounding errors

${ }^{2}$ Among the "other" category included Native American $(n=5)$, Arab/Middle Eastern $(n=2)$, participant-specified other $(n=8)$, and prefer not to disclose or missing $(n=39)$
Finally, they completed further demographic questions and then were asked to read a debriefing paragraph, including resources on vaccination from the CDC and how to find their state and local health departments.

\section{Measures}

\section{Vaccination Status}

Participants were asked, "has [child name] ever received a vaccination?" Those who said no to this question were classified as "never vaccination" $(n=122)$. Those who said yes were then asked, "did you choose to follow the full recommended vaccination schedule for [child name] as appropriate for his or her current age?" and were provided the response options "[child name] received some but not all of the recommended vaccinations" (classified as "partial vaccination", $n=103)$; "[child name] received all the vaccinations on a delayed schedule" (classified as "delayed vaccination", $n=134)$; and "[child name] received all the vaccinations on the standard CDC schedule" (classified as "complete vaccination", $n=420$ ).

\section{Consulting a Pediatrician}

Participants were asked, "Did you consult your pediatrician or family health care provider about your vaccination decisions?".

\section{Following Their Pediatrician's Advice}

Participants who said "yes" to consulting their pediatrician were then asked, "Did you follow the recommendation of your pediatrician or family healthcare practitioner?".

\section{Other Information Sources}

Participants were asked who they turn to for help in making vaccination decisions. Aside from a pediatrician, other sources of information participants could indicate included a spouse or partner, parents, other family members, and friends; participants could select all that applied.

\section{Child Responses to Vaccines}

Participants who indicated that their child had received at least one vaccine $(n=657)$ were asked whether their child had experienced or displayed any of the following reactions to their most recent vaccine, on a scale from 1 (not at all) to 5 (a great deal): crying, general upset, fever, rash, telling me with words that they do not like getting shots. Responses were averaged to create a scale of previous child responses (Cronbach $\alpha=0.81$ ). 


\section{Change to Intention to Vaccinate}

Participants were asked the extent to which the same five child responses to vaccines would influence their likelihood of vaccinating their child in the future, on a scale from 1 (much less likely to vaccinate) to 5 (much more likely to vaccinate); 3 indicated "would not affect likelihood of vaccination" (Cronbach $\alpha=0.89$ ).

\section{Results}

\section{Descriptives and Demographics}

Multinomial logistic regression was used to test whether demographic variables were significantly related to vaccination status. Full statistical results can be found in Table 2 . Income, insurance status, and education level significantly predicted the likelihood of complete vaccination compared to the other vaccination categories. Specifically, as income increased, the likelihood of never vaccinating decreased in comparison to complete vaccination. Having insurance was also associated with a lower likelihood of never compared to complete vaccination. As education level increased the likelihood of delayed vaccination decreased in comparison to complete vaccination. As child age increased, participants were significantly more likely to partially than never vaccinate.

With non-Hispanic white as the comparison group, nonHispanic Black participants reported a higher likelihood of never vaccinating compared to complete vaccination, and a higher likelihood of never vaccinating compared to partial. Non-Hispanic Black participants were also more likely to delay vaccination compared to complete vaccination. Hispanic participants reported a higher likelihood of delayed vaccination compared to complete vaccination, and a higher likelihood of delayed vaccination compared to partial vaccination.

\section{Sources of Information}

Logistic regressions tested for group differences in likelihood of consulting different sources about vaccine decisions. Full statistical results can be found in Table 3. Consulting a pediatrician was significantly less likely for never vaccination compared to all three other groups. There were no differences between the other three groups in likelihood of consulting a pediatrician. Of the 577 participants who said they did consult a pediatrician about their decision, the complete vaccination group was significantly more likely than all three

Table 2 Relationship between demographic variables and vaccination status (multinomial logistic regression with base comparison group varied; comparison group listed first in "group vs. group" notation)

\begin{tabular}{|c|c|c|c|c|c|c|c|c|c|c|c|c|}
\hline & \multicolumn{2}{|c|}{$\begin{array}{l}\text { Complete vs. } \\
\text { delayed }\end{array}$} & \multicolumn{2}{|c|}{$\begin{array}{l}\text { Complete vs. } \\
\text { partial }\end{array}$} & \multicolumn{2}{|c|}{$\begin{array}{l}\text { Complete vs. } \\
\text { none }\end{array}$} & \multicolumn{2}{|c|}{$\begin{array}{l}\text { Delayed vs. } \\
\text { partial }\end{array}$} & \multicolumn{2}{|c|}{ Delayed vs. none } & \multicolumn{2}{|c|}{ partial vs. none } \\
\hline & $\mathrm{rr}$ & $95 \% \mathrm{CI}$ & $\mathrm{rr}$ & $95 \% \mathrm{CI}$ & $\mathrm{rr}$ & $95 \% \mathrm{CI}$ & $\mathrm{rr}$ & $95 \% \mathrm{CI}$ & $\mathrm{rr}$ & $95 \% \mathrm{CI}$ & $\mathrm{rr}$ & $95 \% \mathrm{CI}$ \\
\hline Child age & 0.99 & $0.94,1.03$ & 1.04 & $0.99,1.09$ & 0.96 & $0.91,1.01$ & 1.05 & $0.99,1.12$ & 0.97 & $0.92,1.03$ & 0.92 & $0.87,0.98$ \\
\hline \multicolumn{13}{|l|}{ Child sex } \\
\hline Female & 1.15 & $0.74,1.79$ & 1.27 & $0.79,2.03$ & 1.09 & $0.67,1.77$ & 1.13 & $0.64,1.98$ & 0.97 & $0.55,1.71$ & 0.86 & $0.47,1.56$ \\
\hline Other & 2.33 & $0.44,12.33$ & 0.40 & $0.03,4.91$ & 1.17 & $0.24,5.69$ & 0.18 & $0.01,2.90$ & 0.52 & $0.07,3.68$ & 2.88 & $0.19,42.80$ \\
\hline Respondent age & 0.98 & $0.96,1.01$ & 1.00 & $0.97,1.02$ & 0.99 & $0.96,1.01$ & 1.01 & $0.98,1.04$ & 1.00 & $0.97,1.03$ & 0.99 & $0.96,1.02$ \\
\hline \multicolumn{13}{|l|}{ Respondent sex } \\
\hline Female & 0.81 & $0.47,1.40$ & 1.58 & $0.81,3.08$ & 0.95 & $0.51,1.77$ & 2.06 & $0.96,4.45$ & 1.24 & $0.61,2.53$ & 0.60 & $0.26,1.37$ \\
\hline Other & 0.18 & $0.02,1.32$ & 1.01 & $0.15,6.86$ & 1.72 & $0.39,7.69$ & 6.12 & $0.45,82.52$ & 10.40 & $1.08,99.45$ & 1.70 & $0.19,14.96$ \\
\hline Education & 0.83 & $0.71,0.98$ & 0.89 & $0.74,1.07$ & 0.94 & $0.78,1.12$ & 1.06 & $0.85,1.32$ & 1.11 & $0.90,1.38$ & 1.05 & $0.84,1.32$ \\
\hline Income & 0.95 & $0.86,1.06$ & 0.91 & $0.81,1.02$ & 0.85 & $0.75,0.96$ & 0.96 & $0.83,1.11$ & 0.90 & $0.78,1.04$ & 0.93 & $0.80,1.09$ \\
\hline Insured (yes $=1$ ) & 0.97 & $0.45,2.09$ & 0.64 & $0.30,1.34$ & 0.48 & $0.24,0.96$ & 0.63 & $0.26,1.53$ & 0.47 & $0.20,1.09$ & 0.75 & $0.32,1.74$ \\
\hline Exemption state $($ yes $=1$ ) & 1.51 & $0.97,2.36$ & 1.38 & $0.85,2.23$ & 1.01 & $0.62,1.64$ & 0.91 & $0.51,1.63$ & 0.67 & $0.38,1.19$ & 0.73 & $0.40,1.35$ \\
\hline \multicolumn{13}{|l|}{ Respondent racelethnicity } \\
\hline Black & 2.19 & $1.06,4.54$ & 1.63 & $0.72,3.70$ & 4.00 & $2.00,7.98$ & 0.74 & $0.30,1.85$ & 1.82 & $0.83,4.03$ & 2.45 & $1.02,5.92$ \\
\hline Hispanic/Latino & 2.97 & $1.38,6.41$ & 0.67 & $0.19,2.39$ & 1.09 & $0.37,3.22$ & 0.22 & $0.06,0.84$ & 0.37 & $0.12,1.13$ & 1.64 & $0.36,7.48$ \\
\hline Asian/Pac. Isl & 1.20 & $0.49,2.95$ & 0.74 & $0.24,2.26$ & 1.45 & $0.59,3.60$ & 0.62 & $0.16,2.30$ & 1.21 & $0.39,3.79$ & 1.97 & $0.53,7.31$ \\
\hline Multiracial & 1.35 & $0.49,3.72$ & 1.73 & $0.62,4.82$ & 1.46 & $0.49,4.39$ & 1.28 & $0.38,4.34$ & 1.08 & $0.30,3.87$ & 0.85 & $0.23,3.07$ \\
\hline Other/no answer & 1.76 & $0.51,6.09$ & 1.37 & $0.34,5.50$ & 0.93 & $0.19,4.62$ & 0.78 & $0.16,3.83$ & 0.53 & $0.09,3.14$ & 0.68 & $0.10,4.50$ \\
\hline
\end{tabular}

$\mathrm{rr}=$ relative risk ratio; coefficients significant at $\mathrm{p}<.05$ are bolded for ease of interpretation. Male is the comparison group for analyses with sex, and non-Hispanic white is the comparison group for analyses with racial and ethnic identity 
Table 3 Relationship between vaccination status and demographic variables on use of different sources of vaccine information

\begin{tabular}{|c|c|c|c|c|c|c|c|c|c|c|c|c|}
\hline & \multicolumn{2}{|c|}{ Pediatrician } & \multicolumn{2}{|c|}{$\begin{array}{l}\text { Followed Ped. } \\
\text { Advice }(n=498)\end{array}$} & \multicolumn{2}{|c|}{ Spouse/Partner } & \multicolumn{2}{|c|}{ Parents } & \multicolumn{2}{|c|}{ Other Family } & \multicolumn{2}{|c|}{ Friends } \\
\hline & or & $95 \%$ CI & or & $95 \% \mathrm{CI}$ & or & $95 \%$ CI & or & $95 \% \mathrm{CI}$ & or & $95 \% \mathrm{CI}$ & or & $95 \% \mathrm{CI}$ \\
\hline \multicolumn{13}{|l|}{ Vaccination status } \\
\hline Complete vs. delayed & 0.83 & $0.49,1.41$ & 0.08 & $0.03,0.24$ & 0.87 & $0.54,1.40$ & 1.41 & $0.82,2.42$ & 1.56 & $0.72,3.41$ & 2.04 & $0.98,4.22$ \\
\hline Complete vs. partial & 0.84 & $0.48,1.47$ & 0.02 & $0.01,0.06$ & 0.83 & $0.50,1.39$ & 1.10 & $0.59,2.04$ & 1.94 & $0.88,4.26$ & 3.99 & $2.04,7.82$ \\
\hline Complete vs. none & 0.35 & $0.21,0.58$ & 0.02 & $0.01,0.07$ & 0.73 & $0.44,1.23$ & 1.92 & $1.10,3.34$ & 2.47 & $1.13,5.39$ & 2.29 & $1.07,4.88$ \\
\hline Delayed vs. partial & 1.01 & $0.51,1.99$ & 0.23 & $0.10,0.55$ & 0.96 & $0.52,1.77$ & 0.78 & $0.38,1.58$ & 1.24 & $0.49,3.14$ & 1.96 & $0.89,4.31$ \\
\hline Delayed vs. none & 0.42 & $0.23,0.79$ & 0.28 & $0.11,0.70$ & 0.84 & $0.46,1.55$ & 1.36 & $0.72,2.58$ & 1.58 & $0.63,3.95$ & 1.12 & $0.47,2.65$ \\
\hline Partial vs. none & 0.42 & $0.22,0.81$ & 1.21 & $0.51,2.88$ & 0.88 & $0.46,1.66$ & 1.75 & $0.86,3.56$ & 1.27 & $0.50,3.21$ & 0.57 & $0.25,1.30$ \\
\hline Child age & 1.05 & $1.01,1.09$ & 1.02 & $0.96,1.10$ & 0.94 & $0.91,0.97$ & 1.04 & $0.99,1.08$ & 0.92 & $0.86,0.98$ & 0.98 & $0.93,1.04$ \\
\hline \multicolumn{13}{|l|}{ Child sex } \\
\hline Female & 1.11 & $0.76,1.61$ & 0.57 & $0.30,1.11$ & 0.91 & $0.64,1.29$ & 0.85 & $0.56,1.27$ & 0.92 & $0.52,1.62$ & 0.94 & $0.55,1.59$ \\
\hline Other & 3.19 & $0.51,19.86$ & - & - & 0.46 & $0.13,1.61$ & 1.05 & $0.21,5.09$ & 0.88 & $0.11,6.92$ & 2.53 & $0.35,18.25$ \\
\hline Respondent age & 0.99 & $0.97,1.00$ & 1.01 & $0.98,1.04$ & 1.00 & $0.98,1.02$ & 0.95 & $0.93,0.97$ & 1.07 & $1.04,1.09$ & 1.01 & $0.98,1.04$ \\
\hline \multicolumn{13}{|l|}{ Respondent sex } \\
\hline Female & 0.65 & $0.39,1.06$ & 0.44 & $0.17,1.13$ & 1.06 & $0.68,1.66$ & 0.80 & $0.48,1.32$ & 2.85 & $1.16,7.00$ & 1.85 & $0.91,3.77$ \\
\hline Other & 0.78 & $0.18,3.27$ & 0.06 & $0.01,0.54$ & 1.07 & $0.32,3.56$ & 0.58 & $0.12,2.86$ & 2.04 & $0.30,13.78$ & 0.42 & $0.03,5.03$ \\
\hline Education & 1.10 & $0.95,1.26$ & 0.89 & $0.68,1.17$ & 1.12 & $0.98,1.29$ & 0.86 & $0.73,0.99$ & 0.92 & $0.74,1.14$ & 1.31 & $1.07,1.60$ \\
\hline Income & 0.98 & $0.90,1.07$ & 0.88 & $0.75,1.04$ & 1.14 & $1.04,1.25$ & 0.98 & $0.89,1.09$ & 0.96 & $0.84,1.10$ & 0.92 & $0.80,1.04$ \\
\hline Insured $($ yes $=1$ ) & 1.46 & $0.81,2.65$ & 0.51 & $0.15,1.74$ & 1.86 & $1.09,3.20$ & 0.84 & $0.46,1.53$ & 0.86 & $0.34,2.15$ & 0.54 & $0.25,1.18$ \\
\hline Exemption state $($ yes $=1$ ) & 1.05 & $0.72,1.54$ & 0.65 & $0.33,1.29$ & 1.27 & $0.89,1.80$ & 0.62 & $0.41,0.93$ & 1.10 & $0.61,1.96$ & 1.13 & $0.66,1.93$ \\
\hline \multicolumn{13}{|l|}{ Respondent race/ethnicity } \\
\hline Black & 0.85 & $0.46,1.57$ & 2.74 & $0.81,9.25$ & 0.47 & $0.27,0.82$ & 1.32 & $0.72,2.44$ & 0.83 & $0.29,2.35$ & 0.60 & $0.23,1.59$ \\
\hline Hispanic/Latino & 1.41 & $0.58,3.43$ & 0.75 & $0.20,2.81$ & 1.44 & $0.66,3.18$ & 0.47 & $0.18,1.21$ & 1.17 & $0.33,4.20$ & 0.40 & $0.09,1.79$ \\
\hline Asian/Pac. Isl & 0.87 & $0.42,1.77$ & 1.97 & $0.49,7.97$ & 0.90 & $0.43,1.84$ & 1.17 & $0.54,2.50$ & 1.78 & $0.62,5.14$ & 0.86 & $0.30,2.46$ \\
\hline Multiracial & 1.10 & $0.44,2.73$ & 1.14 & $0.25,5.10$ & 0.71 & $0.32,1.55$ & 0.93 & $0.37,2.31$ & 0.90 & $0.19,4.18$ & 2.45 & $0.92,6.52$ \\
\hline Other/no answer & 2.65 & $0.55,12.78$ & 0.63 & $0.10,4.06$ & 0.86 & $0.31,2.44$ & 1.59 & $0.51,4.90$ & 0.82 & $0.12,5.38$ & 0.49 & $0.06,3.96$ \\
\hline
\end{tabular}

or $=$ odds ratio for dichotomous outcomes where no $=0$ and yes $=1$; coefficients significant at $p<0.05$ are bolded for ease of interpretation. Male is the comparison group for analyses with sex, and non-Hispanic white is the comparison group for analyses with racial and ethnic identity; in comparisons of vaccine status groups the comparison group is listed first in "group vs. group" notation. "-" indicates no participants in the cell for that analysis

other groups to say they followed their pediatrician's advice on vaccinating. The delayed vaccination group, while less likely to say they listened to their pediatrician than the complete vaccination group, were significantly more likely to say they listened than the never vaccination group and than the partial vaccination group. There was no significant difference between never and partial vaccination in their likelihood of reporting that they listened to their pediatrician.

Alternative information sources aside from a pediatrician were also assessed. There were no significant differences by vaccination status in use of a spouse or partner as information source. The never vaccination group was significantly more likely than the complete vaccination group to report consulting their parents and other family members. There were no other significant differences between the groups on either parents or other family. The never vaccination group were significantly more likely to report relying on friends than the complete vaccination group. The partial vaccination group was also significantly more likely to report relying on friends than the complete vaccination group. There were no other significant differences by vaccination group.

\section{Prior Experiences with Vaccines}

Participants who reported their child had received at least one vaccination $(n=657)$ were asked about their adverse reactions to the most recent vaccine, and how much those adverse reactions would change their intention to vaccinate in the future. Ordinary Least Squares (OLS) regression was used to test for group differences, and the child's age at their most recent vaccine was added as a covariate (correlation between age at last vaccine and adverse reactions Pearson's $r=-0.33, p<0.001)$. Full statistical results can be found in Table 4 . Both partial vaccination and delayed vaccination reported higher adverse reactions than did complete vaccination. There was no significant difference between partial 
Table 4 Relationship between vaccination status and demographic variables on prior adverse reactions with vaccines and change in future vaccination intention (for those who have ever received a vaccine, $n=657$ ); coefficients significant at $p<.05$ are bolded for ease of interpretation

\begin{tabular}{|c|c|c|c|c|}
\hline & \multicolumn{2}{|c|}{ Adverse reactions } & \multicolumn{2}{|c|}{$\begin{array}{l}\text { Change in intention to vac- } \\
\text { cinate }\end{array}$} \\
\hline & $b$ & $95 \% \mathrm{CI}$ & $b$ & $95 \% \mathrm{CI}$ \\
\hline \multicolumn{5}{|l|}{ Vaccination status } \\
\hline Complete vs. delayed & 0.48 & $0.26,0.70$ & -0.05 & $-0.22,0.12$ \\
\hline Complete vs. partial & 0.35 & $0.12,0.59$ & -0.45 & $-0.63,-0.27$ \\
\hline Delayed vs. partial & -0.13 & $-0.41,0.15$ & -0.40 & $-0.62,-0.18$ \\
\hline Child age & 0.04 & $0.01,0.06$ & -0.00 & $-0.02,0.02$ \\
\hline Child age at last vaccination & -0.09 & $-0.12,-0.06$ & 0.00 & $-0.02,0.02$ \\
\hline \multicolumn{5}{|l|}{ Child sex } \\
\hline Female & 0.10 & $-0.06,0.26$ & -0.08 & $-0.21,0.05$ \\
\hline Other & -0.14 & $-0.87,0.59$ & 0.03 & $-0.53,0.60$ \\
\hline Respondent age & -0.01 & $-0.02,-0.00$ & -0.00 & $-0.01,0.00$ \\
\hline \multicolumn{5}{|l|}{ Respondent sex } \\
\hline Female & -0.17 & $-0.37,0.04$ & -0.06 & $-0.22,0.10$ \\
\hline Other & 0.50 & $-0.18,1.17$ & -0.11 & $-0.63,0.41$ \\
\hline Education & -0.01 & $-0.07,0.05$ & 0.01 & $-0.04,0.06$ \\
\hline Income & 0.00 & $-0.03,0.04$ & -0.00 & $-0.03,0.03$ \\
\hline Insured (yes $=1$ ) & 0.29 & $-0.01,0.59$ & 0.12 & $-0.13,0.35$ \\
\hline Exemption state $($ yes $=1$ ) & -0.14 & $-0.31,0.02$ & -0.13 & $-0.26,-0.00$ \\
\hline \multicolumn{5}{|l|}{ Respondent race/ethnicity } \\
\hline Black & 0.09 & $-0.22,0.40$ & 0.07 & $-0.17,0.31$ \\
\hline Hispanic/Latino & 0.27 & $-0.08,0.62$ & 0.13 & $-0.14,0.40$ \\
\hline Asian/Pac. Isl & 0.50 & $0.17,0.82$ & -0.06 & $-0.31,0.19$ \\
\hline Multiracial & 0.15 & $-0.25,0.54$ & -0.03 & $-0.34,0.27$ \\
\hline Other/no answer & -0.11 & $-0.65,0.42$ & -0.01 & $-0.42,0.41$ \\
\hline
\end{tabular}

Male is the comparison group for analyses with sex, and non-Hispanic white is the comparison group for analyses with racial and ethnic identity; in comparisons of vaccine status groups the comparison group is listed first in "group vs. group" notation. "-" indicates no participants in the cell for that analysis and delayed vaccination. The partial vaccination group also reported significantly lower intention to vaccinate in the future due to expectations of adverse reactions compared to both delayed and complete vaccination. There was no significant difference between delayed and complete vaccination.

\section{Discussion}

This study explored U.S. parents and their vaccination decisions for their children. Given the public health consequences associated with childhood vaccination hesitancy, such as deadly outbreaks of measles and pertussis (Greenlee \& Newton, 2018; Phadke et al., 2016), understanding the differences between these behavioral groups may inform public health campaigns aimed at increasing acceptance of the CDC schedule.

The vaccination behavior groups clearly differ on a variety of demographic, psychographic, and behavioral metrics. Demographically, lower household income and lack of insurance both were associated with increased likelihood of never vaccinating compared to complete vaccination. This result indicates that there remain structural barriers to vaccination, despite existing initiatives to reduce them, that should be addressed as a public health issue. Lower educational attainment was associated with increased likelihood of delayed vaccination compared to complete vaccination. Non-Hispanic Black Americans reported an increased likelihood of never vaccinating or delayed vaccination compared to complete vaccination, and Hispanic Americans had increased likelihood to delay vaccination. This suggests that demographic differences in vaccination acceptance that have been demonstrated in previous research were also replicated here, particularly in regards to socioeconomic status and racial and ethnic identity (Bryant et al., 2006; Hill et al., 2020; Ventola, 2016).

In terms of where different vaccination groups get their information, the never vaccination group was less likely than all three other groups to report they consulted a pediatrician about their decisions. Of the participants who did consult their pediatrician, never vaccination and partial vaccination were less likely than delayed and complete vaccination to 
report that they followed their pediatrician's advice. The delayed vaccination group, while more likely to report following their pediatrician's advice than partial and never vaccination, were less likely to report following their pediatrician's advice than the complete vaccination group.

People often consult other important people in their lives aside from a physician to help them make health decisions (Brashers et al., 2002; Carlsson, 2000; Dutta-Bergman, 2004; Kelly et al., 2010). The never vaccination group were more likely than the other three groups to report relying on family members and friends and sources of information about vaccines. The partial vaccination group also reported relying on friends for information more than the complete vaccination group. This finding indicates that family and friends become an important source of information for those people who are less likely to rely on the advice from their pediatrician.

Finally, partial and delayed vaccination groups both reported stronger adverse child reactions (fever, rash, crying, etc.) to a previous vaccine than complete vaccination. For partial vaccination, the expectation of such adverse reactions more strongly influenced their decisions about whether to vaccinate their child in the future. This finding indicates that there is some difference in the thought processes of partial and delayed vaccination groups when a child experiences an adverse reaction - people who delay vaccinations decide to still vaccinate completely (albeit on the delayed schedule) while people who partially vaccinate may view an adverse reaction as a reason to completely forego some vaccines. Future research should examine this aspect further to understand what factors might drive some parents to delay versus forego future vaccines due to previous child responses.

\section{Practical Implications}

The results of this survey have implications for public health campaigns and healthcare practitioners. First, household income and insurance status were significantly associated with never vaccinating. This finding indicates that some systemic aspects to finances and insurance practices still may be causing a barrier for some people when it comes to following the vaccination schedule, either completely or even in part, despite programs and policies that already exist to ameliorate these issues. Some potential approaches to alleviate these issues could include practitioners offering payment plans for vaccinations, and both practitioners and public health campaigns informing parents about programs available to them such as the Vaccines for Children Program (National Center for Immunization \& Respiratory Diseases, 2016), or health centers and state health departments that use sliding scales for vaccination charges.

Second, public health campaigns should consider ways to harness the importance of family and friends as information sources about vaccines for those who did not vaccinate on the recommended schedule. For parents who are skeptical or leaning against vaccinations for their children, and who may seek advice from family and friends, a campaign to recruit and train people to talk to people in their lives who are vaccine-hesitant might be one effective way to encourage vaccine acceptance (e.g., a campaign based in two step flow theory, Katz, 1957; Soffer, 2021)).

Finally, previous child responses to vaccines mattered for the delayed and especially for partial vaccination groups. Both groups reported their child experienced adverse responses to a greater extent than did the complete vaccination group. The responses included those described as "mild" by the CDC, such as crying, upset, fever, and rash (Prevention, 2019). Possibly, people who planned to follow the recommended schedule were more likely to interpret their child's response as mild than those who were already vaccine-hesitant; however, the partial vaccination group reported that the anticipation of these responses reduced their intention to vaccinate their children in the future. Practitioners may wish to discuss more clearly with parents the kinds of child reactions that are considered normal and minor, and communicate that these reactions should not be considered a reason to forego vaccines in the future. That said, people in these groups may have actually experienced more extreme reactions in their children than those in the complete vaccination group.

\section{Limitations}

The present study was correlational in nature, meaning that causality cannot be established. While many of the questions imply temporal order (e.g., consulting a pediatrician before making decisions, previous child responses to vaccines), there could be unmeasured third variables influencing these relationships. We were also limited in our ability to conduct further analyses between and within race and ethnicity groups due to small and unequal sample sizes for those groups, and therefore we cannot make claims as to what may be driving the racial differences found in this study. Future research should focus more specifically on these differences in vaccination intentions between different racial and ethnic identities, perhaps by oversampling, especially from groups that are traditionally underrepresented in health research. Future research should also consider qualitative approaches that can gather information about the lived experiences of individuals from marginalized groups in their own words (Carson et al., 2021). Finally, people who did not vaccinate on the standard schedule were more difficult to identify and recruit than were people who were complete vaccinators. Those who were willing to answer a survey about vaccination may systematically differ from those who were unwilling to participate, particularly because previous research 
suggests that never vaccinators tend to be skeptical of science (Goldenberg, 2019). Future work should attempt to find other ways to sample these hesitant participants, such as through trusted community organizations and community leaders. It should be noted that these data were collected before the COVID-19 pandemic, during which vaccination debates have become more strongly polarized. It is possible that post-pandemic beliefs may have changed, and future research should replicate this work in the post-COVID era.

\section{Conclusion}

Vaccine acceptance is a critical public health issue; yet acceptance of the childhood vaccination schedule as recommended by the CDC has decreased in many areas of the United States (Dubé et al., 2013; Ventola, 2016). In addition, routine childhood immunizations decreased during the COVID-19 pandemic (Santoli, 2020). The results of the present study suggest that demographics, sources of information about vaccines, and previous child responses to vaccines are all influential in determining whether a parent chooses no vaccinations, partial vaccinations, delayed vaccinations, or the complete recommended CDC schedule for their child. Public health stakeholders such as governmental organizations, pediatric and family medical care providers, and public health organizations should consider these barriers and facilitators to encourage increased vaccination acceptance.

Supplementary Information The online version contains supplementary material available at https://doi.org/10.1007/s10995-021-03336-8.

Author Contributions ME: conceptualization, data curation, formal analysis, funding acquisition, methodology, project administration, writing - original draft. RA: conceptualization, data curation, methodology, writing - review \& editing. FA: conceptualization, data curation, methodology, writing — review \& editing.

Funding This manuscript was funded in part by a pilot grant from the Health and Risk Communication Center at Michigan State University. The HRCC had no role in the design, analysis, or writing of this article.

\section{Declarations}

Conflict of interest The authors declare that they have no conflict of interests.

Ethical Approval The methods were conducted in accordance with the Declaration of Helsinki and designated exempt by the Institutional Review Board at Michigan State University. Implied informed consent was obtained from all participants in the online survey through the statement "by clicking the 'next' button and continuing to the survey you are consenting to participate in this study."

Consent to Participate All participants provided informed consent.

Consent for Publication N/A.
Availability of Data and Material Data available upon request from the authors.

Code Availability N/A.

\section{References}

Brashers, D. E., Goldsmith, D. J., \& Hsieh, E. (2002). Information seeking and avoiding in health contexts. Human Communication Research, 28(2), 258-271.

Bryant, W. K., Ompad, D. C., Sisco, S., Blaney, S., Glidden, K., Phillips, E., Vlahov, D., Galea, S., \& Project VIVA Intervention Working Group. (2006). Determinants of influenza vaccination in hardto-reach urban populations. Preventive Medicine, 43(1), 60-70.

Carlsson, M. E. (2000). Cancer patients seeking information from sources outside the health care system. Supportive Care in Cancer, 8(6), 453-457.

Carrel, M., \& Bitterman, P. (2015). Personal belief exemptions to vaccination in California: A spatial analysis. Pediatrics, 136(1), 80-88.

Carson, S. L., Casillas, A., Castellon-Lopez, Y., Mansfield, L. N., Barron, J., Ntekume, E., Landovitz, R., Vassar, S. D., Norris, K. C., Dubinett, S. M., \& Nanibaa, G. (2021). COVID-19 vaccine decision-making factors in racial and ethnic minority communities in Los Angeles, California. JAMA Network Open, 4(9), e2127582.

Chung, Y., Schamel, J., Fisher, A., \& Frew, P. M. (2017). Influences on immunization decision-making among US parents of young children. Maternal and Child Health Journal, 21(12), 2178-2187.

Davis, M. M., Ndiaye, S. M., Freed, G. L., Kim, C. S., \& Clark, S. J. (2003). Influence of insurance status and vaccine cost on physicians' administration of pneumococcal conjugate vaccine. Pediatrics, 112(3), 521-526.

de Cantuária Tauil, M., Sato, A. P. S., \& Waldman, E. A. (2016). Factors associated with incomplete or delayed vaccination across countries: A systematic review. Vaccine, 34(24), 2635-2643.

Dempsey, A. F., Schaffer, S., Singer, D., Butchart, A., Davis, M., \& Freed, G. L. (2011). Alternative vaccination schedule preferences among parents of young children. Pediatrics, 128(5), 848-856.

Dubé, E., Laberge, C., Guay, M., Bramadat, P., Roy, R., \& Bettinger, J. A. (2013). Vaccine hesitancy: An overview. Human Vaccines \& Immunotherapeutics, 9(8), 1763-1773.

Dutta-Bergman, M. J. (2004). Primary sources of health information: Comparisons in the domain of health attitudes, health cognitions, and health behaviors. Health Communication, 16(3), 273-288.

Gesser-Edelsburg, A., Walter, N., Shir-Raz, Y., Bar-Lev, O. S., \& Rosenblat, S. (2017). The behind-the-scenes activity of parental decision-making discourse regarding childhood vaccination. American Journal of Infection Control, 45(3), 267-271.

Giambi, C., Fabiani, M., D’Ancona, F., Ferrara, L., Fiacchini, D., Gallo, T., Martinelli, D., Pascucci, M. G., Prato, R., Filia, A., \& Bella, A. (2018). Parental vaccine hesitancy in Italy: Results from a national survey. Vaccine, 36(6), 779-878.

Goldenberg, M. J. (2019). Antivaccination movement exploits public's distrust in scientific authority. $B M J, 367,16960$.

Greenlee, C. J., \& Newton, S. S. (2018). A review of traditional vaccine-preventable diseases and the potential impact on the otolaryngologist. International Archives of Otorhinolaryngology, 22(03), 317-329.

Gust, D. A., Darling, N., Kennedy, A., \& Schwartz, B. (2008). Parents with doubts about vaccines: Which vaccines and reasons why. Pediatrics, 122(4), 718-725.

Hill, H. A., Elam-Evans, L. D., Yankey, D., Singleton, J. A., \& Kang, Y. (2018). Vaccination coverage among children aged 19-35 
months-United States, 2017. Morbidity and Mortality Weekly Report, 67(40), 1123.

Hill, H. A., Yankey, D., Elam-Evans, L. D., Singleton, J. A., Pingali, S. C., \& Santibanez, T. A. (2020). Vaccination coverage by age 24 months among children born in 2016 and 2017-National Immunization Survey-Child, United States, 2017-2019. Morbidity and Mortality Weekly Report, 69(42), 1505.

Katz, E. (1957). The two-step flow of communication: An up-to-date report on an hypothesis. Public Opinion Quarterly, 21(1), 61-78.

Kelly, B., Hornik, R., Romantan, A., Schwartz, J. S., Armstrong, K., DeMichele, A., Fishbein, M., Gray, S., Hull, S., Kim, A., \& Nagler, R. (2010). Cancer information scanning and seeking in the general population. Journal of Health Communication, 15(7), 734-753.

Kurosky, S. K., Davis, K. L., \& Krishnarajah, G. (2016). Completion and compliance of childhood vaccinations in the United States. Vaccine, 34(3), 387-394.

Larson, H. J., Jarrett, C., Eckersberger, E., Smith, D. M., \& Paterson, P. (2014). Understanding vaccine hesitancy around vaccines and vaccination from a global perspective: A systematic review of published literature, 2007-2012. Vaccine, 32(19), 2150-2159.

McKee, C., \& Bohannon, K. (2016). Exploring the reasons behind parental refusal of vaccines. The Journal of Pediatric Pharmacology and Therapeutics, 21(2), 104-109.

Morrison, M., Castro, L. A., \& Meyers, L. A. (2020). Conscientious vaccination exemptions in kindergarten to eighth-grade children across Texas schools from 2012 to 2018: A regression analysis. PLoS Medicine, 17(3), e1003049.

National Center for Immunization and Respiratory Diseases. (2016). About VFC. Retrieved from https://www.cdc.gov/vaccines/progr ams/vfc/about/index.html

Phadke, V. K., Bednarczyk, R. A., Salmon, D. A., \& Omer, S. B. (2016). Association between vaccine refusal and vaccine-preventable diseases in the United States: A review of measles and pertussis. JAMA, 315(11), 1149-1158.

Prevention, C. f. D. C. a. (2019). Your child's first vaccines. Retrieved from https://www.cdc.gov/vaccines/hcp/vis/vis-statements/multi. html
Sadaf, A., Richards, J. L., Glanz, J., Salmon, D. A., \& Omer, S. B. (2013). A systematic review of interventions for reducing parental vaccine refusal and vaccine hesitancy. Vaccine, 31(40), $4293-4304$.

Santoli, J. M. (2020). Effects of the COVID-19 pandemic on routine pediatric vaccine ordering and administration-United States, 2020. MMWR Morbidity and Mortality Weekly Report, 69, 591-593.

Shelby, A., \& Ernst, K. (2013). Story and science: How providers and parents can utilize storytelling to combat anti-vaccine misinformation. Human Vaccines \& Immunotherapeutics, 9(8), 1795-1801.

Smith, P. J., Humiston, S. G., Marcuse, E. K., Zhao, Z., Dorell, C. G., Howes, C., \& Hibbs, B. (2011). Parental delay or refusal of vaccine doses, childhood vaccination coverage at 24 months of age, and the Health Belief Model. Public Health Reports, $126(2$ Suppl), 135-146.

Soffer, O. (2021). Algorithmic personalization and the two-step flow of communication. Communication Theory, 31(3), 297-315.

Temoka, E. (2013). Becoming a vaccine champion: Evidence-based interventions to address the challenges of vaccination. South Dakota Medicine.

Ventola, C. L. (2016). Immunization in the United States: Recommendations, barriers, and measures to improve compliance: Part 1: Childhood vaccinations. Pharmacy and Therapeutics, 41(7), 426.

Yaqub, O., Castle-Clarke, S., Sevdalis, N., \& Chataway, J. (2014). Attitudes to vaccination: A critical review. Social Science \& Medicine, $112,1-11$

Publisher's Note Springer Nature remains neutral with regard to jurisdictional claims in published maps and institutional affiliations. 